\title{
Design of a Loudspeaker Array for Personal Audio in a Car Cabin
}

\author{
XIANGNING LIAO, ${ }^{1}$ JORDAN CHEER, ${ }^{2}$ STEPHEN J. ELLIOTT, ${ }^{2}$ AND SIFA ZHENG ${ }^{1}$ \\ (liaoxiangning@163.com) (j.cheer@soton.ac.uk)（sje@soton.ac.uk)（zsf@tsinghua.edu.cn) \\ ${ }^{1}$ State Key Laboratory of Automotive Safety and Energy, Tsinghua University, Beijing, China \\ ${ }^{2}$ Institute of Sound and Vibration Research, University of Southampton, Southampton, United Kingdom
}

\begin{abstract}
With an increasing interest in personal audio systems, the car cabin is an important environment in which to generate different audio programs in different regions, without the use of headphones. Two algorithms, acoustic contrast control and the least squares method, are considered for producing two independent listening zones, one zone including the front passengers and the other including the rear passengers. The generation of an acceptable acoustic contrast between the front and rear zones, using an array of four standard audio loudspeakers, is limited to relatively low frequencies. In order to provide acceptable control over a larger audio bandwidth, a loudspeaker array mounted on the ceiling of a car cabin is investigated in this paper. A selection process for the configuration of the source array is described. Free field simulations are used to calculate the response of the source array and investigate the performance of the two control algorithms. Since the performance of the least squares method is dependent on the chosen target sound pressures, a method for selecting the target sound pressures is also proposed. Finally, the proposed loudspeaker array is implemented in a real car and the measured results are found to be similar to those predicted from the simulations.
\end{abstract}

\section{O INTRODUCTION}

Personal sound [1], allowing a number of people in one listening environment to listen to different sound programs without the use of headphones, has received great interest since the 1990s. Several approaches, such as acoustic contrast control [2-3], the least squares method [4-5] and planarity control [6-7], have been proposed for generating a clearly audible sound field in a listening zone whilst ensuring that it is inaudible in other zones.

Acoustic contrast control, introduced by Choi and Kim, provides a method of calculating the strengths of a source array that will maximize the ratio between the acoustic potential energy density in the "bright" zone to that in the "dark" zone without considering the phase within the "bright" zone. Acoustic contrast control has been extensively investigated and implemented to generate personal audio systems for different practical cases, such as computer users [3,8], passengers in an aircraft [9], users of mobile devices [10-11], people sitting around a dining table with a parasol [12] and so on. A similar control strategy is described to maximize the energy difference between the "bright" and "dark" zone in [13]. The least squares method, as the main algorithm used in sound field control [14-15], has also been applied in personal audio applications. The least squares method allows the phase of the pressures in the "bright" zone to be controlled to achieve good audio quality in a practical implementation [16-17]. Accordingly, some modified least squares optimization methods for personal audio systems have been proposed, including adding a weighting factor to balance the potential energy in the "dark" zone with the mean square error in the "bright" zone [18], and using the least squares optimization for the "bright" zone with alternative constraints to create acoustic contrast [4]. Coleman and Jackson proposed the planarity control method, which attempts to generate a plane wave in the "bright" zone $[6,19]$ and, therefore, avoids the complications of selecting a target sound field for the bright zone.

The car cabin, as a common listening environment, has received much attention in recent years. A system combining two different loudspeaker array geometries, the four standard car audio loudspeakers for low frequencies and an additional array of eight headrest loudspeakers for high frequencies, has been proposed to provide control over the full audio bandwidth in Ref. [17]. It is concluded that when producing a "bright" zone including the front seats or the rear seats using the four standard car audio loudspeakers, a contrast performance of more than $15 \mathrm{~dB}$ is achieved at frequencies below $200 \mathrm{~Hz}$. At higher frequencies the headrest array is used to create the two listening zones, and although this is effective when generating a "bright" zone in the front seats, when 
producing a listening zone in the rear seats the acoustic contrast is below $10 \mathrm{~dB}$ at some frequencies. Ref. [1] suggests, as a guide, that a level difference of at least $11 \mathrm{~dB}$ between a "bright" zone and the "dark" zones is required to generate a subjectively acceptable personal audio system. More recent work into the subjective aspects of sound zones has demonstrated that the problem is far more complex, as it depends on the program material being reproduced in each zone [20]. Although the results in [20] were perhaps not conclusive because of a difference between how the experienced and inexperienced listeners interpreted the task, they did indicate a potentially higher level of separation being required in certain situations. The lowest level difference acceptable by $50 \%$ of the test subjects was $12.50 \mathrm{~dB}$, while the highest was $31.17 \mathrm{~dB}$. It has also been shown that this situation is further complicated by the relationship between target quality and the level of acceptable interference in the sound zone [21]. Therefore, due to the complexity of the subjective problem, we consider a minimum acoustic contrast requirement of $11 \mathrm{~dB}$ as originally recommended in [1].

In this paper, the performance of a loudspeaker array on the ceiling inside a car cabin is investigated when it is used to generate independent listening zones in the front and rear seats at higher frequencies. A method of designing the geometry and dimensions of this directional ceiling mounted loudspeaker array is presented, along with an investigation into the specification of the target pressures. This loudspeaker array is distinct from that considered in previous work [17] and, coupled with the proposed design method, demonstrates a possible practical approach to generating independent listening zones in the car cabin. In Section 1, two control strategies, acoustic contrast control and the least squares method, are reviewed. Specific methods of selecting the source geometry and of defining the target sound pressure distribution for improving contrast performance are then reported in Section 2. Section 3 describes a practical implementation in a car cabin, using the proposed source array.

\section{CONTROL STRATEGIES}

The practical problem of generating personal audio zones in a car cabin is defined and the two control strategies, acoustic contrast control and the least squares method, are introduced in this section.

The car cabin personal audio system is defined here by two independent listening zones, a front zone encompasses the two front seats and a rear zone encompasses the three rear seats. The interior dimensions of the vehicle are approximately $3 \mathrm{~m} \times 1.8 \mathrm{~m} \times 1.3 \mathrm{~m}$ and a rectangular enclosure with these dimensions, as shown in Fig. 1, is regarded as a simplified representation of the car cabin. The gray region represents the front zone and the black region represents the rear zone. Their specific positions are shown in Table 1.

The vector of complex pressures at $M$ control points at a given frequency in the "bright" zone, $\boldsymbol{p}_{B}$, is related to the vector of complex source strengths, $\boldsymbol{q}$, by the matrix of complex acoustic impedances, $\boldsymbol{Z}_{B}$. Similarly, the vector of complex pressures at $N$ control points in the "dark" zone, $\boldsymbol{p}_{D}$, is equal to $\boldsymbol{Z}_{D} \boldsymbol{q}$, where $\boldsymbol{Z}_{D}$ is the matrix of complex acoustic impedances. That is,

$$
\boldsymbol{p}_{B}=\boldsymbol{Z}_{B} \boldsymbol{q}, \boldsymbol{p}_{D}=\boldsymbol{Z}_{D} \boldsymbol{q}
$$

where $\boldsymbol{q}=\left[\begin{array}{lll}q_{1} & q_{2} & q_{L}\end{array}\right]^{T}, L$ denotes the number of sources in the array. $\boldsymbol{Z}_{B}$ and $\boldsymbol{Z}_{D}$ are the $M \times L$ and $N \times L$ matrices of acoustic transfer impedances from each source to each control point in the "bright" zone and the "dark" zone respectively.

In order to evaluate the performance of the developed personal audio system, three metrics are defined. The main metric is acoustic contrast [2], $C$, which describes the energy difference between the "bright" zone and "dark" zone.

$$
C=\frac{M}{N} \times \frac{\boldsymbol{p}_{B}{ }^{H} \boldsymbol{p}_{B}}{\boldsymbol{p}_{D}{ }^{H} \boldsymbol{p}_{D}}=\frac{M}{N} \times \frac{\boldsymbol{q}^{H} \boldsymbol{Z}_{B}{ }^{H} \boldsymbol{Z}_{B} \boldsymbol{q}}{\boldsymbol{q}^{H} \boldsymbol{Z}_{D}{ }^{H} \boldsymbol{Z}_{D} \boldsymbol{q}} \ldots \ldots
$$

where ${ }^{H}$ is the complex conjugate transpose.

Table 1. The specific positions of the two control zone

\begin{tabular}{llll}
\hline \hline Control Zones & Length, $\mathrm{m}$ & Width, m & Height, m \\
\hline Front zone & 1 to1.2 & 0.2 to 1.6 & 0.8 to 1 \\
Rear zone & 2 to2.2 & 0.2 to 1.6 & 0.8 to 1 \\
\hline
\end{tabular}

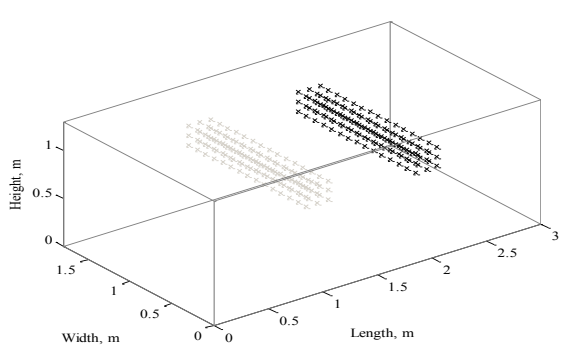

Fig. 1. Two listening zones in a simplified car cabin.

The second metric is the normalized array effort, $A E$, which is given by

$$
A E=\frac{\boldsymbol{q}^{H} \boldsymbol{q}}{\boldsymbol{q}_{r e f}^{H} \boldsymbol{q}_{r e f}}
$$

where $\boldsymbol{q}^{H} \boldsymbol{q}$ is the array effort required by the optimized source array and $\boldsymbol{q}_{r e f}^{H} \boldsymbol{q}_{\text {ref }}$, is the array effort required when the array sources are driven in-phase to produce the same average sound pressure level in the "bright" zone as the personal audio optimized array.

The third index is the normalized error performance, $E r r$, defined as the sum of squared errors between the 
target pressures, $\boldsymbol{p}_{B T}$, and the pressures produced by the source array in the "bright" zone, divided by the sum of the squared target pressures.

$$
E r r=\frac{\left(\boldsymbol{p}_{B T}-\boldsymbol{p}_{B}\right)^{H}\left(\boldsymbol{p}_{B T}-\boldsymbol{p}_{B}\right)}{\boldsymbol{p}_{B T}^{H} \boldsymbol{p}_{B T}}
$$

\subsection{Acoustic Contrast Control}

Acoustic contrast control can be formulated in a few ways [22] to maximize the acoustic contrast performance, $C$. The most logical optimization is expressed as minimizing $\boldsymbol{p}_{D}{ }^{H} \boldsymbol{p}_{D}$ while $\boldsymbol{p}_{B}{ }^{H} \boldsymbol{p}_{B}$ is held constant with a value of $b$. In many practical applications, the electrical power for each individual loudspeaker in the array should also be limited separately. This constraint can be imposed by ensuing that the squared source strength of the $l_{t h}$ source, $\left|q_{l}\right|^{2}$, is below a value of $e_{l}$.

The Lagrangian cost function for this problem can be expressed as:

$$
\begin{aligned}
L= & \boldsymbol{p}_{D}{ }^{H} \boldsymbol{p}_{D}+\lambda_{B}\left(\boldsymbol{p}_{B}{ }^{H} \boldsymbol{p}_{B}-b\right)+\ldots \\
& \sum_{l=1}^{L} \lambda_{l}\left(\left|q_{l}\right|^{2}-e_{l}\right)
\end{aligned}
$$

where $\lambda_{B}$ is the real Lagrange multiplier governing the constraint on the bright zone acoustic energy and $\lambda_{l}$ is the real and positive Lagrange multiplier governing the effort constraint on the $l_{t h}$ source.

By differentiating the cost function with respect to $\boldsymbol{q}$, equating this to zero, and rearranging gives:

$$
\left(\boldsymbol{Z}_{D}{ }^{H} \boldsymbol{Z}_{D}+\lambda_{\boldsymbol{L}}\right)^{-1} \boldsymbol{Z}_{B}{ }^{H} \boldsymbol{Z}_{B} \boldsymbol{q}=-\frac{1}{\lambda_{B}} \boldsymbol{q}
$$

where $\lambda_{L}$ is a diagonal matrix of $L$ Lagrange multipliers governing the $L$ source effort constraints.

From Eq. (6), the optimal solution for $\boldsymbol{q}$ is proportional to the eigenvector corresponding to the largest eigenvalue of $\left(\boldsymbol{Z}_{D}{ }^{H} \boldsymbol{Z}_{D}+\lambda_{\boldsymbol{L}}\right)^{-1} \boldsymbol{Z}_{B}{ }^{H} \boldsymbol{Z}_{B}$.

\subsection{The Least Squares Method}

The least squares approach for generating a personal audio system attempts to minimize the error between the target pressures, $\boldsymbol{p}_{B T}$, and the sound pressures produced by the source array in the "bright" zone, whilst the sum of the squared pressures in the "dark" zone, $\boldsymbol{p}_{D}{ }^{H} \boldsymbol{p}_{D}$, is also minimized. Additionally, by constraining the effort for each source separately, the cost function for this problem can be expressed as:

$$
\begin{aligned}
L= & \left(\boldsymbol{p}_{B T}-\boldsymbol{p}_{B}\right)^{H}\left(\boldsymbol{p}_{B T}-\boldsymbol{p}_{B}\right)+\boldsymbol{p}_{D}{ }^{H} \boldsymbol{p}_{D}+\ldots \\
& \sum_{l=1}^{L} \lambda_{l}\left(\left|q_{l}\right|^{2}-e_{l}\right)
\end{aligned}
$$

For the condition where the number of control points is greater than the number of sources, the source strength vector that minimizes the cost function in $\mathrm{Eq}$. (7) is obtained as:

$$
\boldsymbol{q}=\left[\boldsymbol{Z}_{B}{ }^{H} \boldsymbol{Z}_{B}+\boldsymbol{Z}_{D}{ }^{H} \boldsymbol{Z}_{D}+\lambda_{\boldsymbol{L}}\right]^{-1} \boldsymbol{Z}_{B}{ }^{H} \boldsymbol{p}_{B T}
$$

\section{SELECTION OF THE LOUDSPEAKER ARRAY}

In order to achieve good personal audio at frequencies above $200 \mathrm{~Hz}$, the performance of a number of different ceiling-mounted source array configurations have been investigated. It should be highlighted that the contributions from the room and the effects of objects in close proximity to the sources may have a significant effect on the performance of the loudspeaker array. Although these effects could be modeled using numerical methods, the computational complexity would be high and it would therefore be difficult to conveniently investigate a number of control geometries. Therefore, these effects will be neglected in this investigation. Based on the approach taken in previous work [17], we have assumed that the response is dominated by the direct sound field and, therefore, used a free-field model to conduct the following investigation of loudspeaker geometries. Although this assumption may be limited for some of the geometries investigated here, it provides a useful engineering guide and this is confirmed in Section 3 by the similarity between the free field predictions and the experimental results. Aside from these modelling considerations, it is also worth highlighting that, due to the complexities and uncertainties of the real car cabin, it will not be possible in practice to reliably compensate for the reverberant sound field using the loudspeaker array and, therefore, we focus on controlling the direct field.

In practical applications, loudspeakers have a finite size, which results in an increasing directivity at higher frequencies. To simulate this effect, each source in the following simulations is made up of a group of monopoles all driven in phase. The directivity patterns of finite-sized sources simulated with different numbers of monopoles are compared in Appendix A and based on these results, a $0.08 \mathrm{~m}$ diameter loudspeaker is simulated using a group of 11 monopoles.

It is worth mentioning that in the following investigation, the loudspeaker array geometries have been compared using the acoustic contrast and array effort, while the normalized error has been omitted for brevity.

\subsection{Selection of source array locations}

Firstly, an 8-source array is considered, which is divided into two parts, with each part consisting of four sources, as shown in Fig. 2. The spacing between each source in each part of the source array in the $y$-direction is held constant at a value of $0.1 \mathrm{~m}$. The distance between the two parts of 
the array in the $x$-direction is varied to be $1 \mathrm{~m}, 0.8 \mathrm{~m}, 0.6 \mathrm{~m}$, $0.4 \mathrm{~m}, 0.2 \mathrm{~m}$ and $0.1 \mathrm{~m}$, as shown in Fig. 2. Due to the symmetry of the source configuration in Fig. 2 with respect to the two listening zones, the control performance for either a front "bright" zone or a rear "bright" zone is the same, so only the condition for a front "bright" zone is discussed in the following discussion.

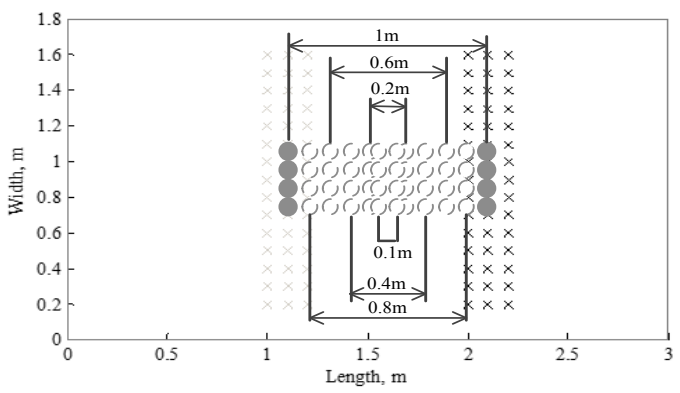

Fig. 2. Different distances between the two parts of the source array. (Gray circles represent sources.)

Based on the six source configurations shown in Fig. 2, the control performance achieved using acoustic contrast control and the least squares method, plotted in decibels, is shown in Fig. 3, optimized to generate a front "bright" zone without an array effort constraint. The results for acoustic contrast control, shown in Fig. 3(a-b), provide an upper bound on the performance for a given geometry. It can be seen that very high levels of contrast performance can, in principle, be achieved below about $1 \mathrm{kHz}$, but only with very high levels of control effort. Additionally, it should be reiterated that the acoustic contrast method puts no constraint on the phase of the pressures in the bright

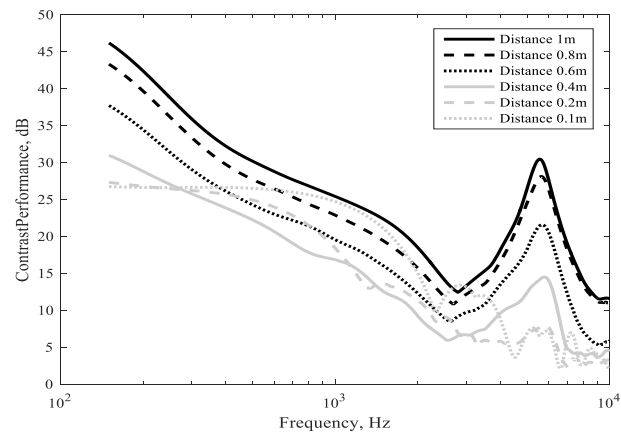

(a) Acoustic contrast optimized contrast performance

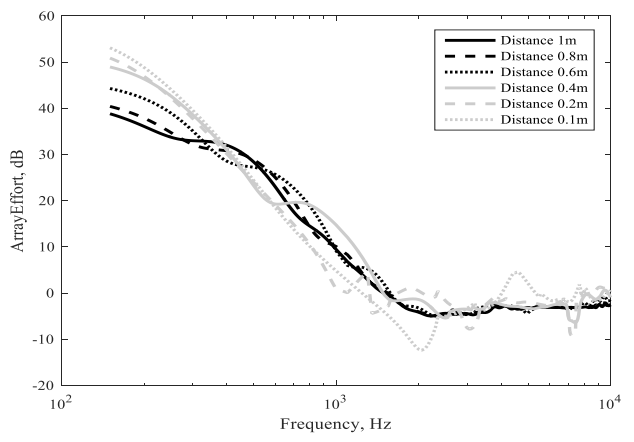

(b) Acoustic contrast optimized array effort zone and, therefore, is generally reported to produce poor audio quality. It is possible to overcome these issues by employing the least squares method, which also controls the phase of the pressures in the bright zone by defining a target sound field. In this case, the vector of target pressures, $\boldsymbol{p}_{B T}$, has been defined as the pressures produced when the four loudspeakers closest to the "bright" zone have been driven in-phase. This does not mean that the pressures in the "bright" zone are all inphase, but that the pressures have the natural spatial response of the near field loudspeakers. The results achieved using the least squares method are shown in Fig. 3 (c-d). Below about $2 \mathrm{kHz}$, the contrast performance for a separation of $0.1 \mathrm{~m}$ is much better than for the other array configurations, but this condition needs a higher array effort. The performance achieved when using the least squares method is different from that achieved when using acoustic contrast control. This is because the least squares method is not only influenced by the geometry of the source array and listening zones but also depends on the target pressures. Above about $2 \mathrm{kHz}$, when the distance between the two parts of the source array is larger, the contrast levels are similar to the results for acoustic contrast control, as this performance results from the natural directivities of the source array.

Summarizing the results presented in Fig. 3 (c-d), it can be concluded that below $2 \mathrm{kHz}$, the highest performance is achieved when the two parts of the source array are separated by $0.1 \mathrm{~m}$. However, above $2 \mathrm{kHz}$, the highest performance is achieved when the two parts of the source array are separated by $1 \mathrm{~m}$, i.e., directly located above the listening zones.

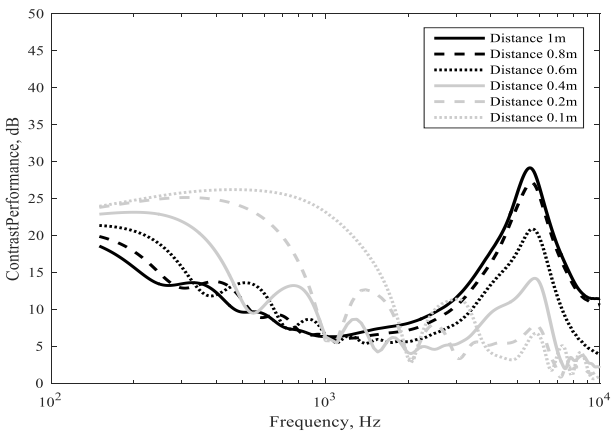

(c) Least squares optimized contrast performance

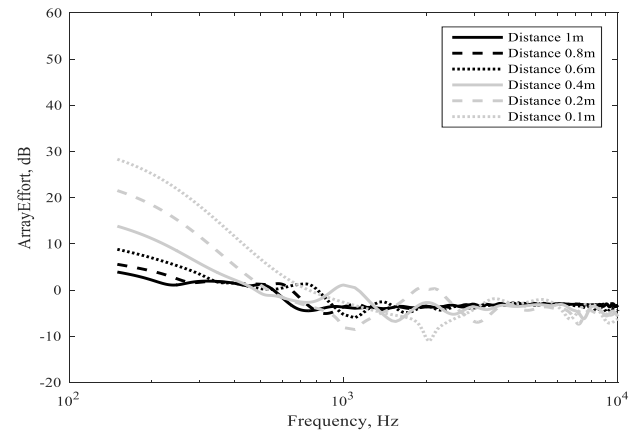

(d) Least squares optimized array effort

Fig. 3. Performance, plotted in decibels, as a function of frequency for the array configurations shown in Fig. 2 optimized using acoustic contrast control (a-b) and the least squares method (c-d), with the target pressures in the "bright" zone defined as those produced by driving the four sources that are closest to the "bright" zone in-phase. 
Based on these observations, a new configuration, shown in Fig. 4, is proposed. The eight sources are now divided into 4 pairs. Using the symmetry of the arrangement, each of the two sources with the same position in the $x$-direction are combined to form a pair. The distance in the $x$-direction between the inner pairs is $0.1 \mathrm{~m}$ and the distance between the outer pairs is $1 \mathrm{~m}$. In the following discussion, the influence of the spacing between the sources in each pair in Fig. 4 will be investigated. As a first step, the spacing between the sources in the outer pairs is held constant to be $0.1 \mathrm{~m}$, and the spacing between the sources in the inner pairs is varied, as shown in Fig. 5.

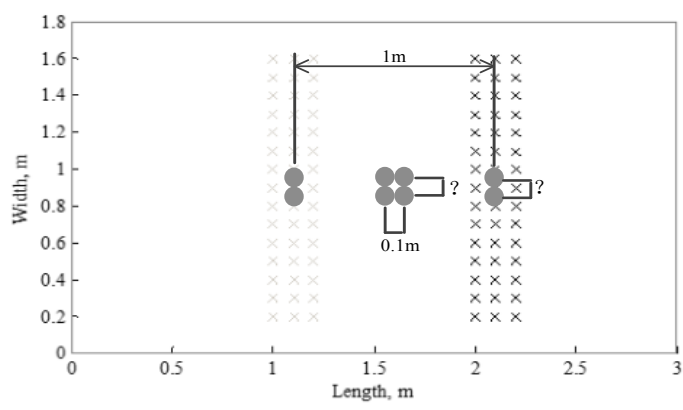

Fig. 4. The basic geometry of an eight source array.

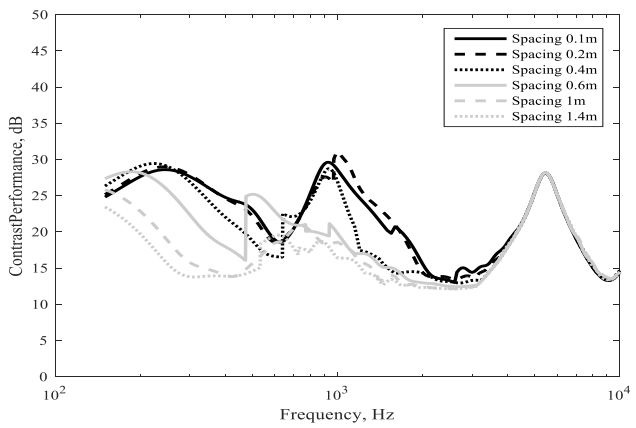

(a) Acoustic contrast optimized contrast performance

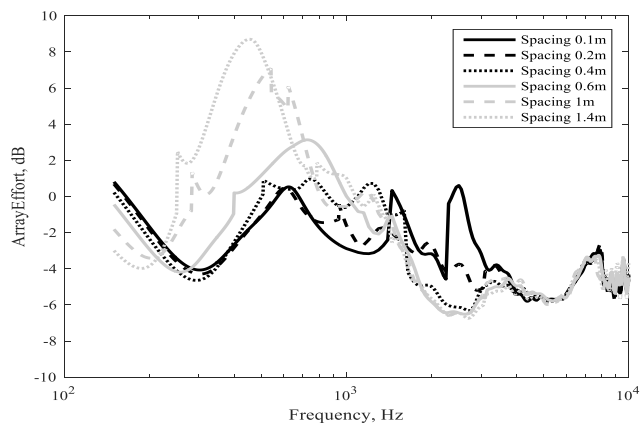

(b) Acoustic contrast optimized array effort
Fig. 6 present the performance of the source geometry in Fig. 5 when it is optimized using two control methods with an appropriate degree of regularization provided by $\lambda_{L}$ in Eq. (6) and (8). It can be seen that no matter for acoustic contrast control or the least squares method, the performance for the array with a spacing between the inner source pairs of $0.1 \mathrm{~m}$ and $0.2 \mathrm{~m}$ is similar, and better than the performance for the other geometries. This indicates that a spacing between the sources in the inner pairs of no more than $0.2 \mathrm{~m}$ is appropriate.

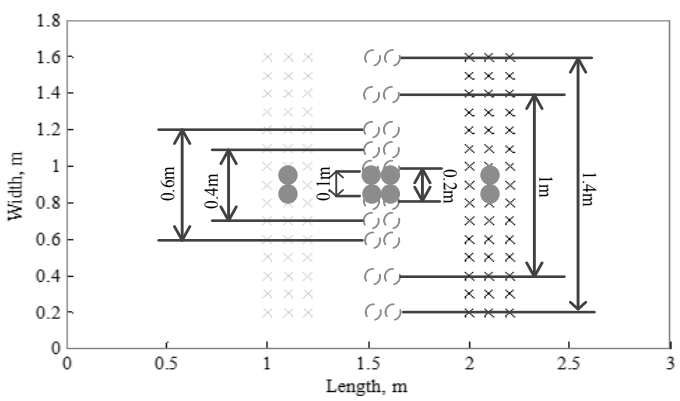

Fig. 5. Different spacing between the sources in the inner pairs.

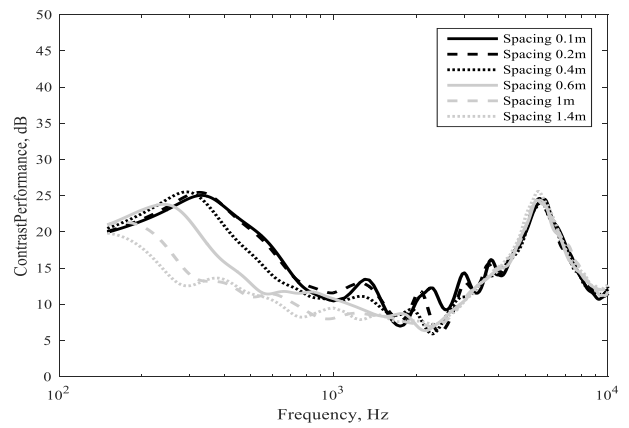

(c) Least squares optimized contrast performance

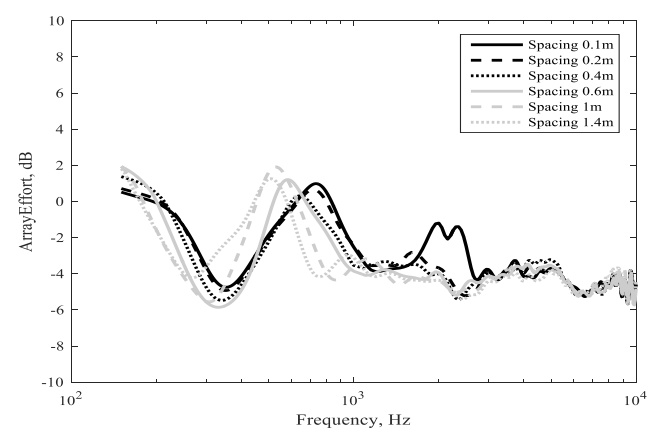

(d) Least squares optimized array effort

Fig. 6. Performance, plotted in decibels, as a function of frequency for the array configurations shown in Fig. 5 optimized using acoustic contrast control (a-b) and the least squares method (c-d), with the target pressures in the "bright" zone defined as those produced by driving the four sources that are closest to the "bright" zone in-phase.

With the spacing of the sources in the inner pairs fixed at $0.1 \mathrm{~m}$, the spacing of the outer pairs has been varied to be $0.1 \mathrm{~m}, 0.2 \mathrm{~m}, 0.4 \mathrm{~m}, 0.6 \mathrm{~m}, 1 \mathrm{~m}$ and $1.4 \mathrm{~m}$, as shown in Fig. 7. The corresponding performance is shown in Fig. 8 for the acoustic contrast and least squares optimizations.

From Fig. 8 (a-b), it can be seen that for acoustic contrast control, the difference between the contrast performance for the different source geometries is smaller than that observed for the inner source pairs. In Fig. 8(c- d), it can be seen that using the least squares method, the bandwidth over which $15 \mathrm{~dB}$ of contrast is achieved is the widest for the source array with a spacing of $0.4 \mathrm{~m}$. This corresponds to an even distribution of the sources within the listening zone, which is $1.2 \mathrm{~m}$ long and, therefore, provides a useful guide for the design of practical systems in which the size of the listening zones may be modified by the seating arrangement. 


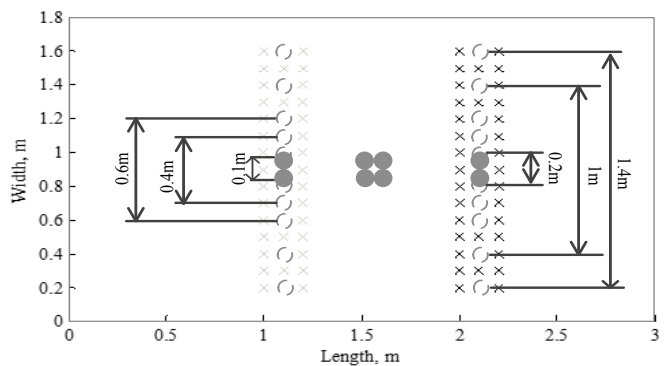

Fig. 7. Different spacing between the sources in the outer pairs.

Based on the results presented in this section, Fig. 9 shows the final source array geometry selected for generating personal sound zones above $200 \mathrm{~Hz}$. It should be highlighted that although the array configuration shown in Fig. 9 is 'suboptimal' when using the acoustic contrast

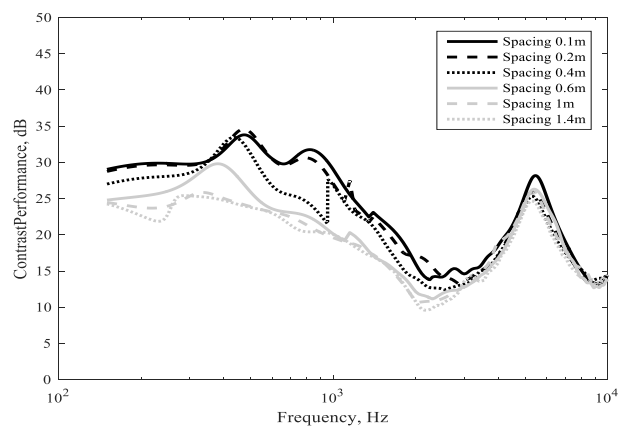

(a) Acoustic contrast optimized contrast performance

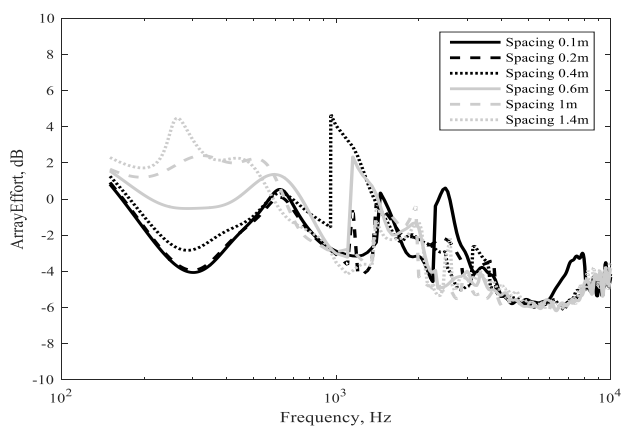

(b) Acoustic contrast optimized array effort control strategy, it in fact achieves the highest average acoustic contrast over frequencies when using the least squares optimization method.

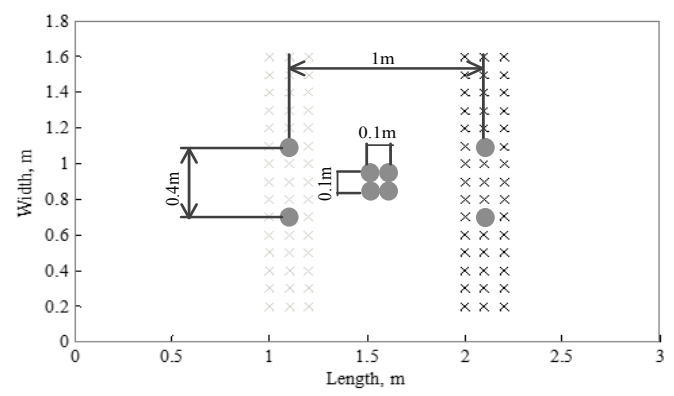

Fig. 9. The source array configuration selected based on the results in Section 2.1.

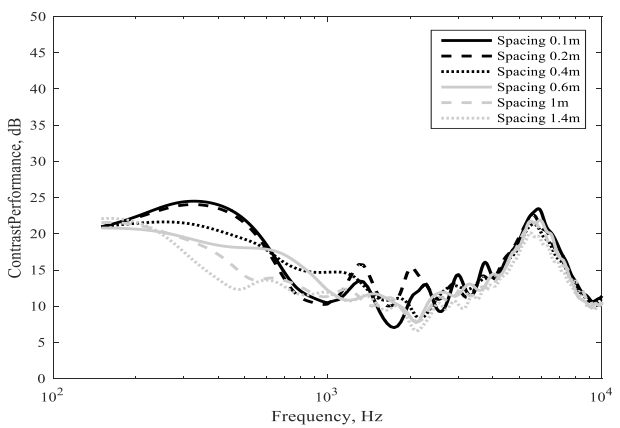

(c) Least squares optimized contrast performance

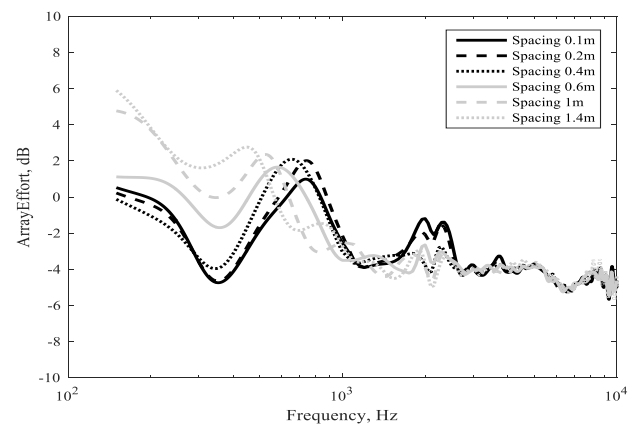

(d) Least squares optimized array effort

Fig. 8. Performance, plotted in decibels, as a function of frequency for the array configurations shown in Fig. 5 optimized using acoustic contrast control (a-b) and the least squares method (c-d), with the target pressures in the "bright" zone defined as those produced by driving the four sources that are closest to the "bright" zone in-phase.

\subsection{Selection of the target sound pressures}

Acoustic contrast control achieves higher levels of contrast compared to the least squares method, but the least squares method is generally expected to provide better audio quality within the listening zones. It has been emphasized in Ref. [16] that with appropriate target sound pressures, the least squares method can provide a contrast performance close to that obtained by acoustic contrast control. Additionally, it has been shown that the least squares method is less susceptible to the uncertainties that will occur in a practical system than acoustic contrast control in [22]. Based on the results presented in the above discussion, the least squares method is a more useful optimization method for practical applications.
In order to improve the contrast performance achieved by the least squares method, three different target pressure scenarios are investigated for the proposed source configuration shown in Fig. 9. The three target pressures are explained in Table 2 and the resulting acoustic contrast, array effort and normalized error are shown in Fig. 10. It can be seen from Fig. 10 that below about $1.8 \mathrm{kHz}$, the contrast performance achieved when using target pressures 3 is mainly greater than $15 \mathrm{~dB}$. Above $2 \mathrm{kHz}$, a higher contrast performance is achieved when using target pressures 2. Comparing these results with the contrast performance shown in Fig. 3(c), it can be concluded that below about $1.8 \mathrm{kHz}$, the inner pairs of the source array make the main contribution to personal audio control, so using the corresponding target sound pressures produced 
by the inner pairs of the source array can achieve a better contrast performance. Above $2 \mathrm{kHz}$, the outer pairs of the source array make the main contribution to the personal audio control, so using the corresponding target sound pressures 2 can provide a larger contrast level between the listening zones.

Table 2. Description of the three target pressures.

\begin{tabular}{ll}
\hline \hline Target Pressure & Description \\
\hline $\begin{array}{l}\text { Target Pressure } \\
1\end{array}$ & $\begin{array}{l}\text { Produced by driving the four sources that } \\
\text { are closest to the "bright" zone in-phase. }\end{array}$ \\
Target Pressure & $\begin{array}{l}\text { Produced by driving the two sources in } \\
\text { the outer pair that are closest to the } \\
2\end{array}$ \\
"bright" zone in-phase. \\
$\begin{array}{l}\text { Target Pressure } \\
3\end{array}$ & $\begin{array}{l}\text { Produced by driving the two sources in } \\
\text { the inner pair that are closest to the } \\
\text { "bright" zone in-phase. }\end{array}$ \\
\hline
\end{tabular}

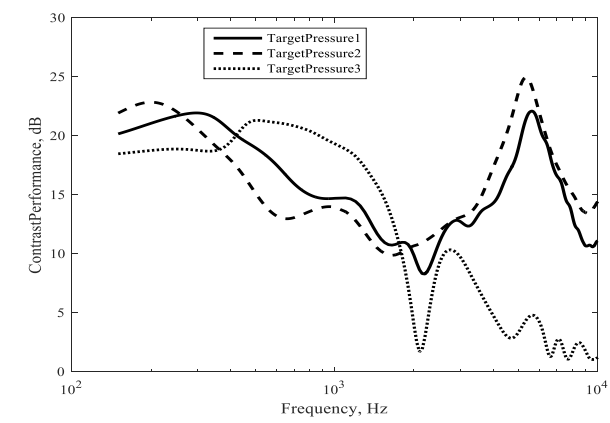

(a) Contrast performance

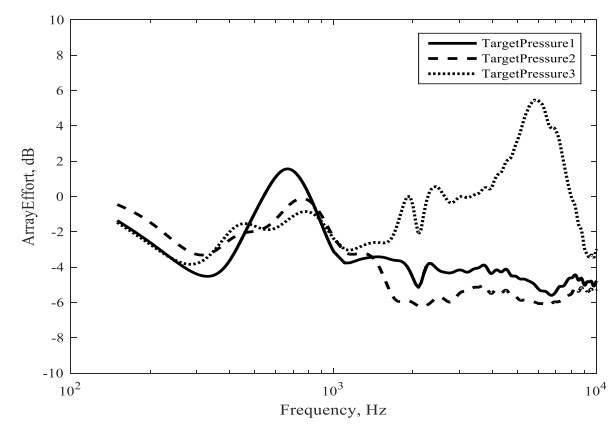

(b) Array effort

Fig. 10. Performance, plotted in decibels, as a function of frequency for the array configuration shown in Fig. 12 optimized using the least squares method with the target pressures in the "bright" zone defined according to Table 2.

Based on the preceding observations, a new target pressure distribution is proposed to achieve a higher level of acoustic contrast performance for the proposed source array in Section 2.1 using the least squares method. Below $2 \mathrm{kHz}$, the outer pair of sources that are closest to the "bright" zone is used to define the target pressures. Above $2 \mathrm{kHz}$, the inner pair of sources that are closest to the "bright" zone is used to define the target pressures. Based on this definition of the target pressures, the contrast performance for the proposed source array geometry is greater than $11 \mathrm{~dB}$ up to $10 \mathrm{kHz}$, as shown in Fig. 11 .

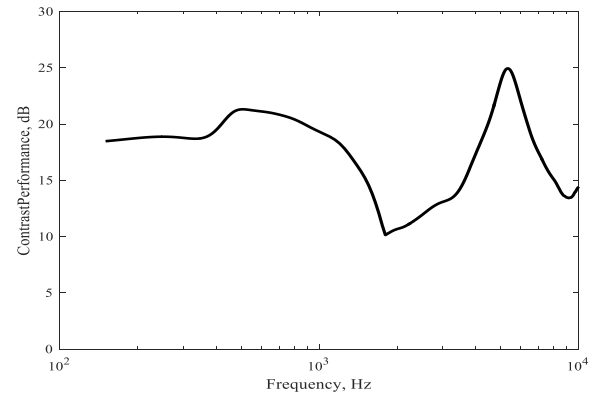

(a) Contrast performance

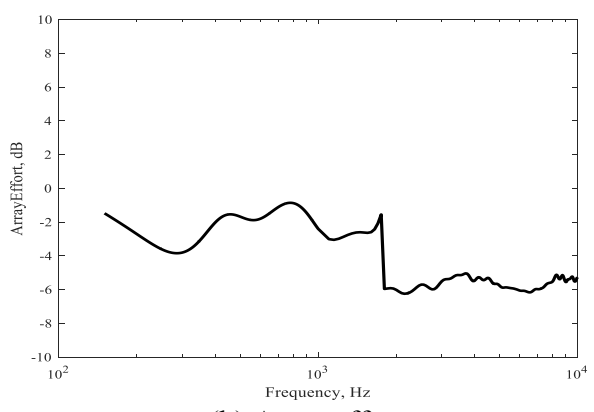

(b) Array effort

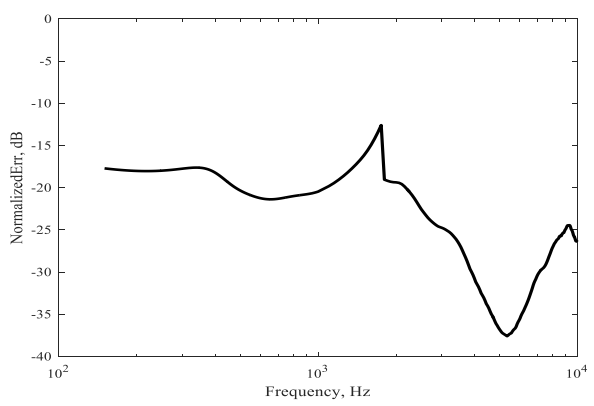

(c) Normalized error

Fig. 11. Performance, plotted in decibels, as a function of frequency for the array configurations shown in Fig. 9 optimized using the least squares method with the proposed target pressures definition.

\subsection{Selection of the diameter of the source array}

From the results shown in Fig. 11 it can be seen that between $1.8-3.5 \mathrm{kHz}$, the contrast performance is still less than $15 \mathrm{~dB}$. In section 2.2 , it has been shown that the outer pairs of sources have the most influence over the contrast performance between around $1.8-3.5 \mathrm{kHz}$. Therefore, in an attempt to improve the acoustic contrast within this frequency range, the diameter of the outer pairs of sources in this array has been increased in order to increase their natural directivity. The diameter of the inner pair of sources is kept at $0.08 \mathrm{~m}$. The diameter of the sources in the outer pairs is varied to be $0.12 \mathrm{~m}, 0.14 \mathrm{~m}$ and $0.16 \mathrm{~m}$, which are simulated using groups of 30, 36 and 42 monopoles respectively. The method to choose the number of monopoles is the same as the method for the source with a diameter of $0.08 \mathrm{~m}$.

The control performance for the modified geometries, using the least squares method, is shown in Fig. 12, from which it can be seen that below about $1.5 \mathrm{kHz}$, the contrast performance is almost the same for the different source configurations. This result can be related to the conclusion in Section 2.2, that the sources in the inner pairs make the 
main contribution to contrast control at frequencies below $1.5 \mathrm{kHz}$. Additionally, it is found that at the lower frequencies, the sources are all activated in phase even though the diameter of the individual sources changes. With larger diameter sources in the outer pairs, the contrast performance and the normalized error are improved at higher frequencies, although the array effort is not unduly changed. This result can be explained by the increased directivity for the sources in the frequency bandwidth 1.5$2.5 \mathrm{kHz}$. Based on the above discussion, increasing the diameter of the sources in the outer pairs to increase the natural directivity of the source array is a good idea for improving the contrast control at higher frequencies. However, it should be noted that in these simulations it is assumed that the loudspeaker continues to act as a piston at these relatively high frequencies, whereas in a practical realization this condition is only likely to be approximately met.

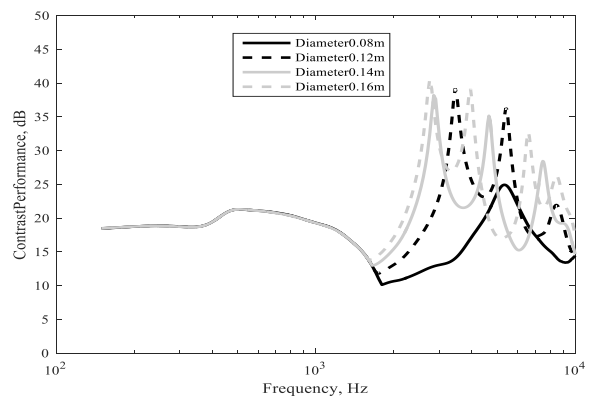

(a) Contrast performance

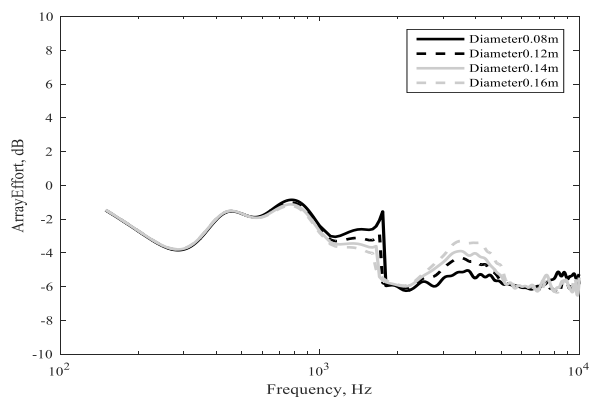

(b) Array effort

Fig. 12. Performance, plotted in decibels, as a function of frequency for the array configuration shown in Fig. 13 optimized using the least squares method with the proposed target pressures definition when the diameters of the sources in the outer pairs are enlarged to be $0.12 \mathrm{~m}, 0.14 \mathrm{~m}, 0.16 \mathrm{~m}$.

\section{EXPERIMENTAL IMPLEMENTATION}

A specific design of source array has been proposed, from the simulations, to produce independent front and rear listening zones at frequencies above $200 \mathrm{~Hz}$. However, these simulations have been conducted in a free field environment and the effects of a practical car cabin are not taken into account. Additionally, the directivities of a source in the above simulations may not be the same as a practical loudspeaker of a given size. Therefore, to validate the predicted performance of the proposed source array for personal audio control above $200 \mathrm{~Hz}$, a source array based on the results of the simulations has been implemented in the car cabin.
Fig. 13(a-b) shows examples of the loudspeaker clusters used to implement the practical array, which is shown in Fig. 13(c). It should be highlighted that the spacing between the outer sources has been increased compared to the geometry shown in Fig. 9, because in the practical implementation the "bright" zone does not span the full region between the seats, which was defined as the "bright" zone in the simulations, but is instead defined by the regions at the two seats. Therefore, as indicated by the simulation based study, the best performance can be achieved when the outer source pairs are evenly distributed across the listening zone and this design guidance has been used here. Because of the symmetrical source configuration, the source strengths of the two loudspeakers in each of the inner pairs should be the same, so these two sources are connected and driven in phase. It was shown in section 2.3 that increasing the diameter of the sources in the outer source pairs can improve the contrast between the listening zones. In the practical setup the larger sources were realized using four single loudspeaker elements, as shown in Fig. 13(b), all driven in phase. An array of 15 microphones, consisting of three rows of five microphones in two planes, was positioned at each seat position in turn, as shown in Fig. 13(d), and the two control zones were therefore defined by 30 microphones in the front and rear zones respectively.

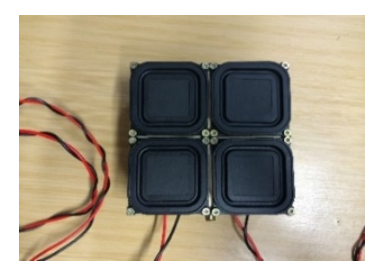

(a)

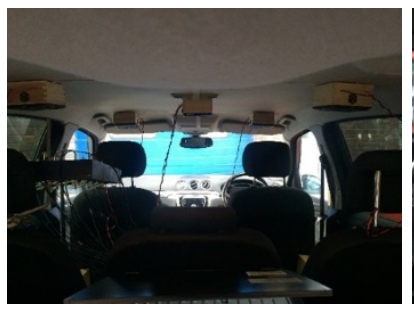

(c)

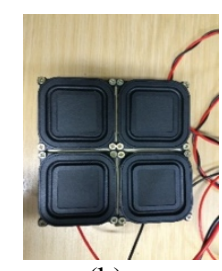

(b)

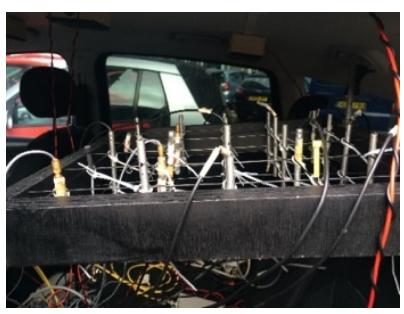

(d)

Fig. 13. The loudspeaker array and microphone setup in the car cabin. (a) Four loudspeakers in the array used for the inner pairs (two loudspeakers in each pair are driven together). (b) Four loudspeakers in the array used for the outer pairs (in which all the loudspeakers are driven in phase). (c) The loudspeaker array on the roof. (d) The microphone array setup in the front seat.

Fig. 14 shows two examples of the impulse responses measured in the car cabin. Fig. 14(a) shows the response measured between the outer loudspeaker array located above the driver and a microphone in the driver's position, whilst Fig. 14(b) shows the response from the inner loudspeaker pair to the same microphone. From Fig. 14(a) it can be seen that, as assumed in Section 2, the response is dominated by the direct path. However, from Fig. 14(b) it can be seen that there is a significant contribution from the indirect sound field due to the distance between the inner loudspeaker pair and the microphone. The indirect paths are not included in the free field simulations 
employed in the previous section and, therefore, it is still important to confirm that the performance of the proposed array in the car cabin is consistent with the simulation results.

The performance of the proposed personal audio system is predicted based on the measured transfer responses between the voltage inputs to each loudspeaker and the outputs of each of the 60 microphones. Fig. 15 shows the control performance calculated from $200 \mathrm{~Hz}$ to $10 \mathrm{kHz}$ using the measured transfer responses for the proposed source array when optimized using the least squares method to produce a front "bright" zone or a rear "bright" zone. It can be seen that using the proposed source array, the predicted acoustic contrast is more than $15 \mathrm{~dB}$ up to 10 $\mathrm{kHz}$ when producing a rear "bright" zone. In the frequency range of $4.5-7 \mathrm{kHz}$, the predicted contrast for the front "bright" zone is a little smaller than that for the rear "bright" zone. In the free field simulations, the two sound zone generation problems were symmetric, whereas they are not in the real car cabin, which gives rise to some differences in the performance for the two cases. The contrast performance decreases near $1 \mathrm{kHz}$ and has a peak at about $4 \mathrm{kHz}$ in both cases, which is consistent with the simulation results in Fig. 11(a).

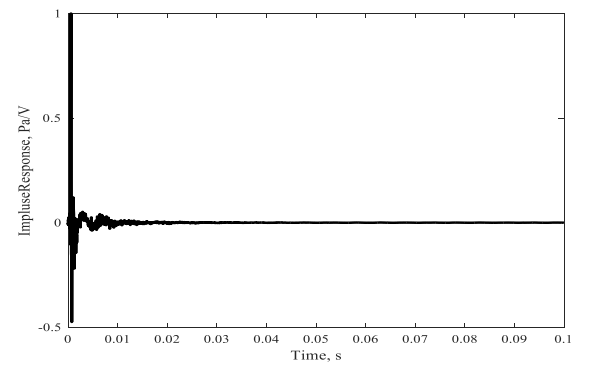

(a) Response from front driver side outer loudspeaker array to a microphone in the driver location.

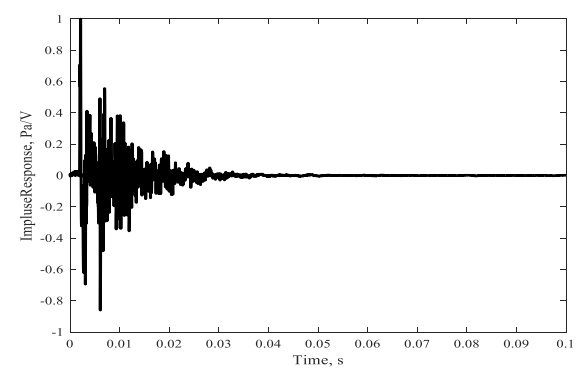

(b) Response from the inner loudspeaker pair to a microphone in the front zone.

Fig. 14. Examples of the impulse responses measured in the car cabin.

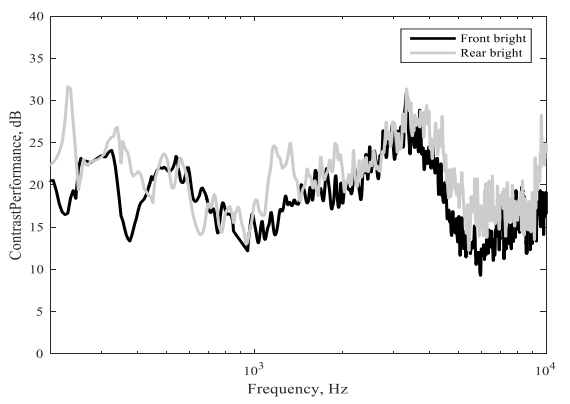

(a) Contrast performance

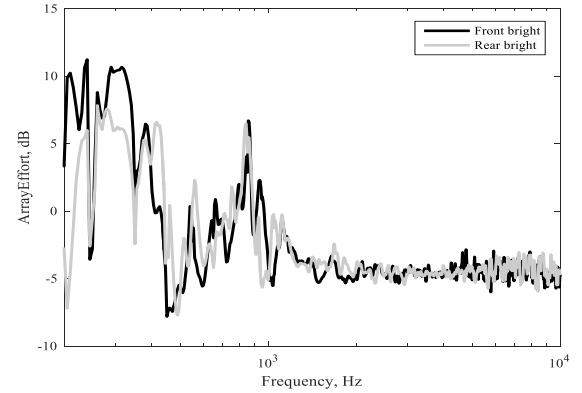

(b) Array effort

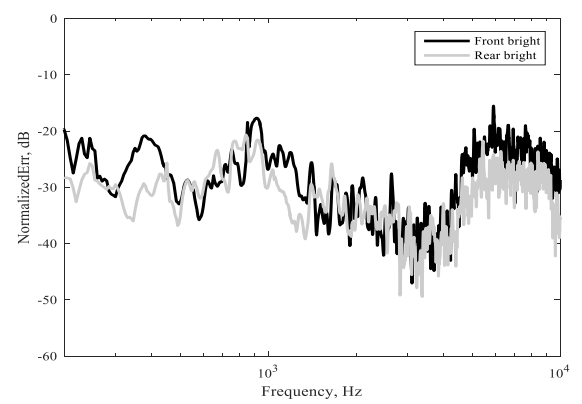

(c) Normalized error

Fig. 15. The performance as a function of frequency predicted using the measured transfer responses for the proposed source array when optimized to produce a front "bright" zone (Thick lines) or a rear "bright" zone (Fine lines) using the least squares method.

Fig. 16 shows the calculated strength of each source in each of the three pairs in the array, plotted in decibels, when optimized to produce a front "bright" zone. Below about $500 \mathrm{~Hz}$, the sources in the inner pairs are driven hardest and are largely in-phase and above $1.5 \mathrm{kHz}$, the sources on the roof above the front listening zone make the main contribution to the contrast control. The levels of the sources in the rear listening zones indicate that these sources do not contribute significantly to the generation of a front "bright" zone. These results are generally consistent with the corresponding theoretical predictions shown in Section 2.3.

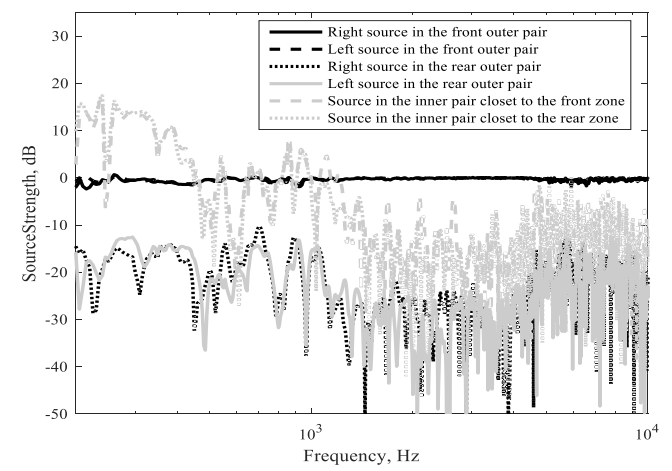

Fig. 16. The source strength for each source when producing a front "bright" zone. (The left or right source labelling corresponds to the geometry in Fig. 9. For example, Right source in the front pair presents the right source in the outer pair on the roof above the front listening zone.) 


\section{CONCLUSIONS}

To achieve independent front and rear listening zones, which the four standard car audio loudspeakers cannot achieve above about $200 \mathrm{~Hz}$, a new type of source array mounted on the ceiling of a car cabin has been investigated. A process of selecting a suitable source configuration is described in detail and the results show that in the frequency range between $200 \mathrm{~Hz}$ and $1 \mathrm{kHz}$, a higher contrast can be achieved when the sources are mounted on the roof between the front and rear zones. At higher frequencies, sources mounted on the ceiling directly above the front and rear zones achieves better performance for personal audio in these positions.

Two optimization strategies, acoustic contrast control and the least squares method, are applied to evaluate the control performance of the proposed source array. As expected, the results show that acoustic contrast control achieves the maximum contrast between the different listening zones, however, it has been highlighted that with suitably selected target pressures, described in Section 2.2, the least squares method can also achieve a good level of contrast control. Additionally, the least squares method can provide an improved audio quality and is more robust to the uncertainties in the practical implementation [17]. Therefore, as is well known, the least squares method is a more suitable method in practice.

To validate the results of the free field simulations, an array with 8 independent sources is implemented in a car cabin. The performance of this array configuration is predicted using the transfer responses measured from the loudspeaker drive signals to the outputs of the microphones in the front and rear listening zones. When this system is optimized to produce a rear "bright" zone, the proposed source array almost achieves a contrast of 15 dB. When generating a front "bright" zone, the performance of the source array is limited in the range of $4.5-7 \mathrm{kHz}$, but is still more than $11 \mathrm{~dB}$ up to $10 \mathrm{kHz}$.

Compared with the car cabin personal audio system reported in [17], the presented system is able to achieve a significant boost in performance in the mid frequency range between 1 and $4 \mathrm{kHz}$. It also achieves more consistent performance when generating either a front or a rear "bright" zone. This is achieved mainly by using the natural directivity of the finite-sized sources, as suggested in [1], in this case positioned on the ceiling of the car cabin.

\section{ACKNOWLEDGEMENT}

This work has been supported by the National Natural Science Foundation of China Project through the grant 51275262.

\section{REFERENCES}

[1] W. F. Druyvesteyn and J. Garas, "Personal Sound," J. Audio Eng. Soc., vol. 45, pp. 685-701(1997 Sep.).

[2] J. W. Choi and Y. H. Kim, "Generation of An Acoustically Bright Zone with an Illuminated Region Using Multiple Sources," J. Acous. Soc. Am., vol. 111, pp. 1695-1700(2002 Apr.).
[3] J. H. Chang, C. H. Lee, J. Y. Park and Y. H. Kim, "A Realization of Sound Focused Personal Audio System Using Acoustic Contrast Control," J. Acous. Soc. Am., vol. 125, pp. 2091-2097 (2009 Apr.).

[4] Y. Cai, M. Wu and J. Yang, "Sound Reproduction in Personal Audio Systems Using the Least-Squares Approach with Acoustic Contrast Control Constraint," J. Acous. Soc. Am., vol. 135, pp. 734-741(2014 Feb.).

[5] M. Shin, F. M. Fazi and P.A. Nelson, "Controlled Sound Field with a Dual Layer Loudspeaker Array," J.Sound Vib., vol. 333, pp. 3794-3817(2014 Apr.).

[6] P. Coleman, P. J. B Jackson, M. Olik, M. Møller, M. Olsen and J. A. Pedersen, "Acoustic Contrast, Planarity and Robustness of Sound Zone Methods Using a Circular Loudspeaker Array," J. Acous. Soc. Am., vol. 135, pp. 1929-1940(2014 Apr.).

[7] P. Coleman, P. J. B Jackson, M. Olik and J.A. Pedersen, "Personal Audio with a Planar Bright Zone," $J$. Acous. Soc. Am., vol. 136, pp. 1725-1735(2014 Aug.).

[8] J. Y. Park, J. H. Chang, and Y. H. Kim, "Generation of Independent Bright Zones for a Two-Channel Private Audio System," J. Audio Eng. Soc., vol. 58, pp. 382393(2010 May).

[9] S. J. Elliott and M. Jones, "An Active Headrest for Personal Audio," J. Acous. Soc. Am., vol. 119, pp. 27022709 (2006 Feb.).

[10] S. J. Elliott, J. Cheer, H. Murfet and K. R. Holland, "Minimally Radiating Sources for Personal Audio," $J$. Acous. Soc. Am., vol. 128, pp. 1721-1728 (2010 Nov.).

[11] J. Cheer, S. J. Elliott, Y. Kim and J.W. Choi, "Practical Implementation of Personal Audio in a Mobile Device," J. Audio Eng. Soc., vol. 61, pp. 290-300 (2013 May).

[12] J. M. Lee, T. W. Lee, J. Y. Park and Y. H. Kim, "Generation of a Private Listening Zone; Acoustic Parasol," presented at the 20th Congress on Acoustics (2010 Aug.).

[13] M. Shin, S. Q. Lee, F. M. Fazi, P.A. Nelson, D. S. Kim, S. Y. Wang, K. H. Park and J. G. Seo, "Maximization of Acoustic Energy Difference Between Two Spaces," J. Acous. Soc. Am., vol. 128, pp. 121-131 (2010 July).

[14] F. M. Fazi, "Sound Field Reproduction", Master's thesis, University of Southampton, UK (2010).

[15] T. Lentz, "Dynamic Crosstalk Cancellation for Binaural Synthesis in Virtual Reality Environments," $J$. Audio Eng. Soc., vol. 54, pp. 283-294 (2006 Apr.).

[16] M. F. S. Gálvez, S. J. Elliott and J. Cheer, "A Superdirective Array of Phase Shift Sources," J. Acous. Soc. Am., vol. 132, pp. 746-756 (2012 June).

[17] J. Cheer, S. J. Elliott and M. F. S. Gálvez, "Design and Implementation of a Car Cabin Personal Audio System," J. Audio Eng. Soc., vol. 61, pp. $412-424$ (2013 June).

[18] J. H. Chang and F. Jacobsen, "Sound Field Control with a Circular Double-Layer Array of Loudspeakers," J. Acous. Soc. Am., vol. 131, pp. 4518-4525 (2012 Apr.).

[19] P. Coleman, P, Jackson, M. Olik and J. A. Pedersen, "Optimizing the Planarity of Sound Zones," AES 52nd International Conference: Sound Field Control-Engineering and Perception (2013 Sep.), conference paper 5-1. 
[20] J. Francombe, R. Mason, M. Dewhirst, and S. Bech "Determining the threshold of acceptability for an interfering audio programme," 132nd AES Convention (2012 Apr.), conference paper 8639 .

[21] K. Baykaner, P. Coleman, R. Mason, P. Jackson, J. Francombe, M. Olik, and S. Bech, "The relationship between target quality and interference in sound zone," $J$. Audio Eng. Soc., vol. 63, pp. 78-89 (2015 Jan).

[22] S. J. Elliott, J. Cheer, J. W. Choi and Y. H. Kim, "Robustness and Regularization of Personal Audio Systems," IEEE Trans. on Aud. Sp. and Lang. Proc., vol. 20, pp. 2123-2133 (2012 Sep.).

\section{APPDENIX A}

For all the simulations in Section 2.1, each source, with a diameter of $0.08 \mathrm{~m}$, has been simulated as a group of 11 single monopole sources, as shown in Fig. A1.

The number of monopoles has been chosen to be sufficient when the directivities of the source no longer vary as the number of employed monopoles is increased.
To assess this criterion, the directivities of a source consisting of a different number of monopoles are compared at $2 \mathrm{kHz}, 4 \mathrm{kHz}$ and $8 \mathrm{kHz}$ in Fig. A2-A4.

From Fig. A2, it can be seen that at frequencies below 2 $\mathrm{kHz}$, no matter how many monopoles are included in the simulations, each source can be approximately identified as a monopole. From Fig. A3-A4, it can be seen that at higher frequencies, with an increasing number of monopoles, the directivity increases, which agrees with the theoretical behavior of a finite-sized source. When the number of monopoles is larger than 11 , the directivities remain largely unchanged. Therefore, a group of 11 monopoles is used to simulate a source with a diameter of $0.08 \mathrm{~m}$.

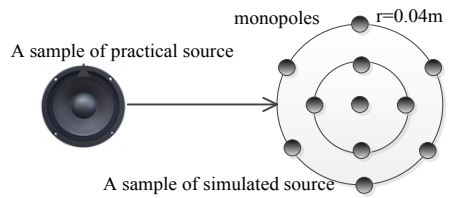

Fig.A1. A finite-sized practical source is simulated as a group of 11 monopole sources driven in-phase.
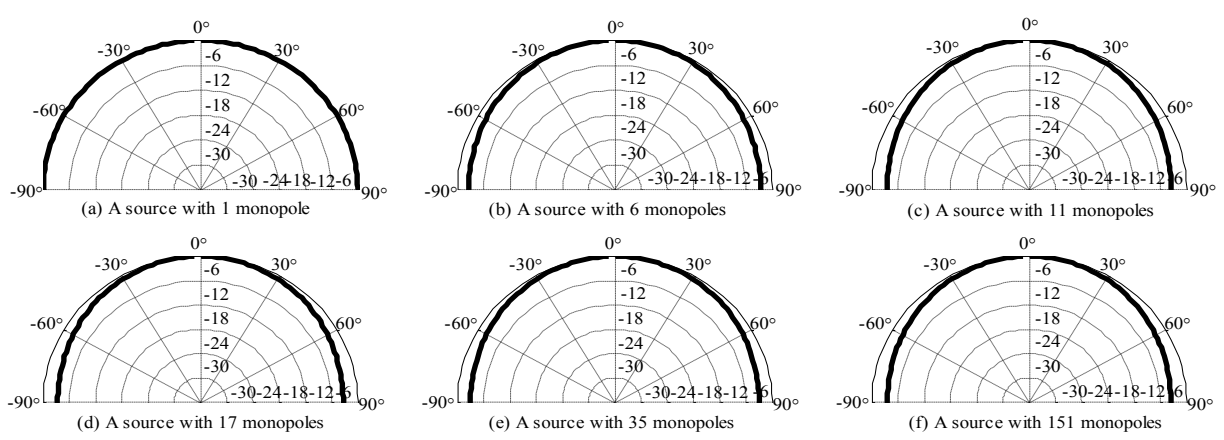

Fig. A2. The directivities of a finite-sized source with different numbers of monopoles used in the simulation at $2 \mathrm{kHz}$.
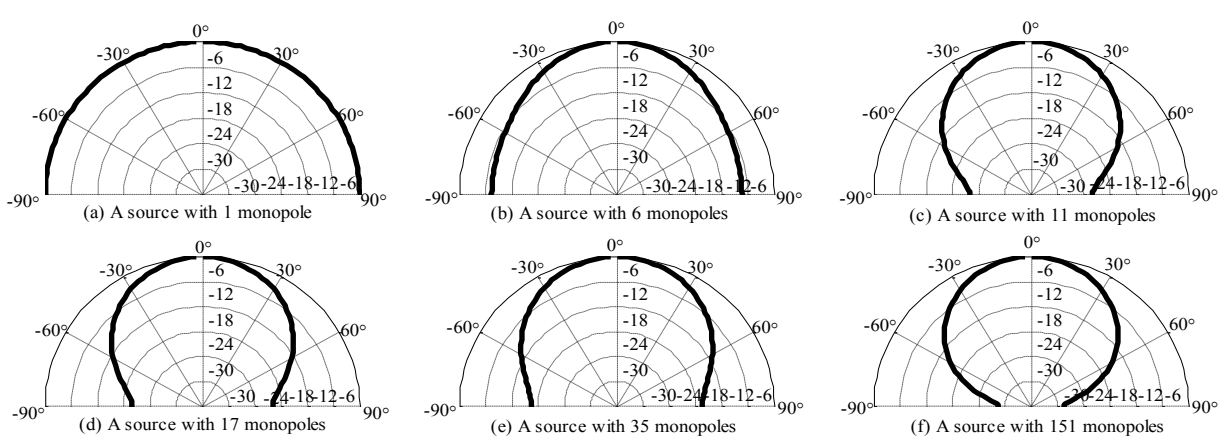

Fig. A3. The directivities of a finite-sized source with different numbers of monopoles used in the simulation at $4 \mathrm{kHz}$.
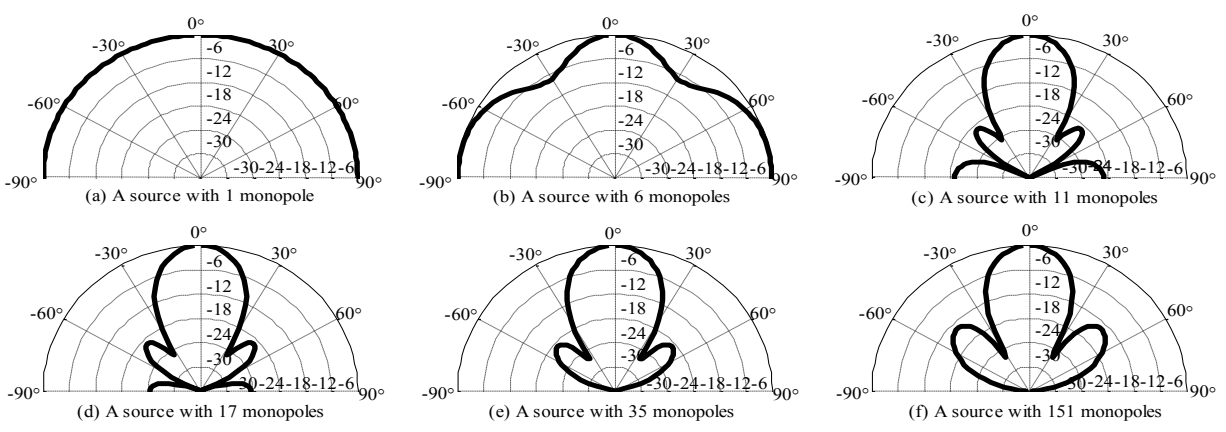

Fig. A4. The directivities of a finite-sized source with different numbers of monopoles used in the simulation at $8 \mathrm{kHz}$. 
THE AUTHORS

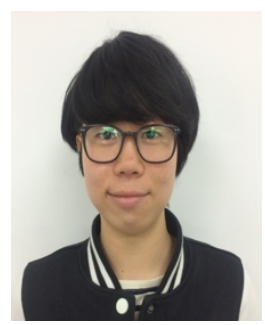

Xiangning Liao

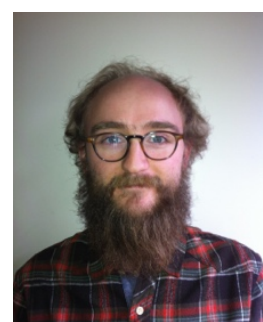

Jordan Cheer
Xiangning Liao received the bachelor's degree in mechanical engineering from Chongqing University in 2012 and in September she came to Tsinghua University for pursuing $\mathrm{Ph}$.D. degree in automotive engineering under the supervision of Professor S.F. Zheng. She works on sound reproduction and active control for personal audio systems. In the learning process, she received the M.S. Long Award and Y.G. Fei Award for academic performance. In the half year between September in 2015 and February in 2016, she went to the Institute of Sound and Vibration Research(ISVR), University of Southampton, as a visiting student and had a cooperation with Professor S. J. Elliott and Research Fellow J. Cheer.

Jordan Cheer graduated with first class honours B.Mus. (Tonmeister) in music and sound recording from the Institute of Sound Recording, University of Surrey, U.K. in 2008, and the M.Sc. and Ph.D. degrees in sound and vibration studies from the Institute of Sound and Vibration Research (ISVR), University of Southampton, U.K., in 2009 and 2012, respectively. He was appointed as a Research Fellow in the ISVR in 2012 and promoted to a Senior Research Fellowship in 2015. He was awarded a New Frontiers Fellowship in 2015 and continues to work in both active control of noise and vibration and array signal processing for audio reproduction. Dr. Cheer received the P. E. Doak Award for academic performance in the taught part of the M.Sc., the E. J. Richards Prize for the best masters dissertation, the Sir James Lighthill Award for the best student paper at the 19th International Congress on Sound and Vibration and the Maureen Mew prize for the best graduating PhD from the ISVR.

Steve Elliott graduated with first class joint honours BSc in physics and electronics from the University of London, in 1976, and received the $\mathrm{PhD}$ degree from the University of Surrey in 1979 for a dissertation on musical acoustics.

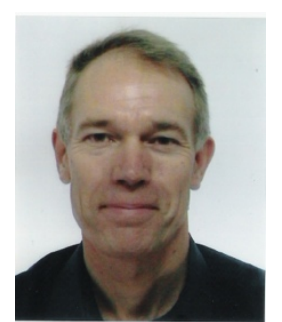

Steve Elliott

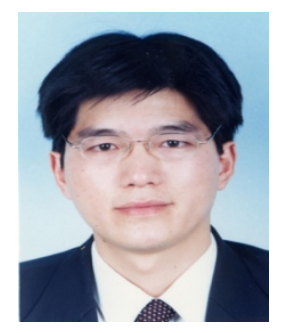

Sifa Zheng
He was appointed Lecturer at the Institute of Sound and Vibration Research (ISVR), University of Southampton, in 1982, was made Senior Lecturer in 1988, Professor in 1994, and served as Director of the ISVR from 2005 to 2010. His research interests have been mostly concerned with the connections between the physical world, signal processing and control, mainly in relation the active control of sound using adaptive filters and the active feedback control of vibration. This work has resulted in the practical demonstration of active control in propeller aircraft, cars and helicopters. His current research interests include modular systems for active feedback control and modelling the active processes within the cochlear.

Professor Elliott has published over 250 papers in refereed journals and 500 conference papers and is coauthor of Active Control of Sound (with P.A. Nelson 1992), Active Control of Vibration (with C.R. Fuller and P.A. Nelson 1996) and author of Signal Processing for Active Control (2001). He is a Fellow of the Acoustical Society of America, was jointly awarded the Tyndall Medal from the Institute of Acoustics in 1992 and the Kenneth Harris James Prize from the Institution of Mechanical Engineers in 2000. He was made a Fellow of the Royal Academy of Engineering in 2009.

Sifa Zheng graduated with joint honors in automotive engineering and electronics from Tsinghua University in 1993 and received the Ph.D. degree from Tsinghua University in 1997. After a period as a lecturer at the department of Automotive Engineering at the Tsinghua University, he was made associate professor in 2003, doctorial supervisor in 2010 and professor in 2014. He has published over 100 papers and is an editor member of ACTA ACUSTICA, (Chinese version), Chinese Journal of Acoustics (English version) and senior member of Chinese Mechanical Engineering Society. 